\title{
Stereotactic radiosurgery for idiopathic glossopharyngeal neuralgia: an international multicenter study
}

\author{
Hideyuki Kano, MD, PhD, ${ }^{1}$ Dusan Urgosik, MD, ${ }^{2}$ Roman Liscak, MD, ${ }^{2}$ Bruce E. Pollock, MD, ${ }^{3}$ \\ Or Cohen-Inbar, MD, PhD, ${ }^{4,7}$ Jason P. Sheehan, MD, PhD, ${ }^{4}$ Mayur Sharma, MD, ${ }^{5}$ Danilo Silva, MD, ${ }^{5}$ \\ Gene H. Barnett, MD, ${ }^{5}$ David Mathieu, MD, ${ }^{6}$ Nathaniel D. Sisterson, BA, ${ }^{1}$ and L. Dade Lunsford, MD1 \\ 1'Department of Neurological Surgery, Center for Image-Guided Neurosurgery, University of Pittsburgh, Pennsylvania; \\ 2Stereotactic and Radiation Neurosurgery, Hospital Na Homolce, Prague, Czech Republic; ${ }^{3}$ Department of Neurological Surgery, \\ Mayo Clinic, Rochester, Minnesota; ${ }^{4}$ Department of Neurological Surgery, University of Virginia Health System, Charlottesville, \\ Virginia; ${ }^{5}$ Department of Neurological Surgery, Cleveland Clinic, Cleveland, Ohio; ${ }^{6}$ Université de Sherbrooke, Centre de \\ Recherche du CHUS, Sherbrooke, Quebec, Canada; and 'Department of Neurological Surgery, Rambam Health Care Center, \\ Haifa, Israel
}

\begin{abstract}
OBJECTIVE The goal of this study was to evaluate the outcomes of Gamma Knife stereotactic radiosurgery (SRS) when used for patients with intractable idiopathic glossopharyngeal neuralgia.

METHODS Six participating centers of the International Gamma Knife Research Foundation identified 22 patients who underwent SRS for intractable glossopharyngeal neuralgia between 1998 and 2015. The median patient age was 60 years (range 34-83 years). The median duration of symptoms before SRS was 46 months (range 1-240 months). Three patients had unsuccessful prior surgical procedures, including microvascular decompression (MVD) $(n=2)$ and balloon compression $(n=1)$. The radiosurgical target was the glossopharyngeal meatus. The median maximum dose was $80 \mathrm{~Gy}$. RESULTS The median follow-up was 45 months after SRS (range 6-120 months). Twelve patients (55\%) had $<4$ years of follow-up. Thirteen patients (59\%) had initial complete pain relief at a median of 12 days after SRS (range 1-60 days). Three patients (14\%) had partial pain relief at a median of 70 days after SRS (range 60-90 days). Six patients (27\%) had no pain relief. Among 16 patients with initial pain relief, 5 maintained complete pain relief without medication (Barrow Neurological Institute [BNI] pain intensity score Grade I), 1 maintained occasional pain relief without medication (BNI Grade II), 3 maintained complete pain relief with medication (BNI Grade IIIb), and 7 patients had pain recurrence at a median of 20 months after SRS (range 6-120 months). The rates of maintenance of adequate pain relief (BNI Grades I-IIlb) were $63 \%$ at 1 year, $49 \%$ at 2 years, $38 \%$ at 3 years, $38 \%$ at 5 years, and $28 \%$ at 7 years. When 7 patients without pain recurrence within 4 years of follow-up were excluded, the rates of maintenance of adequate pain relief were $38 \%$ at 5 years and $28 \%$ at 7 years. Ten patients required additional procedures (MVD, $n=4$; repeat SRS, $n=5$; glossopharyngeal nerve block, $n=1$ ). Four of 5 patients who underwent repeat SRS maintained pain relief (BNI Grade I, $n=3$; and BNI Grade Illb, $n=1$ ). No adverse effects of radiation were observed after a single SRS. Two patients developed hyperesthesia in the palatoglossal arch 5 and 8 months after repeat SRS, respectively.
\end{abstract}

CONCLUSIONS Stereotactic radiosurgery for intractable, medically refractory glossopharyngeal neuralgia provided lasting pain reduction in $55 \%$ of patients after 1 or 2 SRS procedures. Patients who had a poor response or pain recurrence may require additional procedures such as repeat SRS, MVD, nerve blocks, or nerve section. No patient developed changes in vocal cord function or swallowing disorders after SRS in this study.

http://thejns.org/doi/abs/10.3171/2016.7.GKS161523

KEY WORDS stereotactic radiosurgery; Gamma Knife; glossopharyngeal neuralgia; facial pain

$\mathrm{G}$ LOSSOPHARYNGEAL neuralgia (GPN) is a rare pain syndrome of the ninth cranial nerve characterized by severe episodic ear, tongue, and throat pain. The pain can be triggered by chewing, swallowing, coughing, and speaking and lasts from a few seconds to a few minutes. In rare cases GPN can be associated with hemodynamic instability such as arrhythmias, hypotension, syncope, and cardiac arrest. ${ }^{1,5}$ GPN can be caused by glossopharyngeal nerve compression by a vessel at the root entry zone of the brainstem. ${ }^{4}$ GPN may also be

ABBREVIATIONS BNI = Barrow Neurological Institute; GPN = glossopharyngeal neuralgia; IGKRF = International Gamma Knife Research Foundation; MVD = microvascular decompression; SRS = stereotactic radiosurgery; $\mathrm{TN}=$ trigeminal neuralgia.

SUBMITTED June 10, 2016. ACCEPTED July 22, 2016.

INCLUDE WHEN CITING DOI: 10.3171/2016.7.GKS161523. 
secondary to tumors, aneurysms, infections, and multiple sclerosis. ${ }^{4}$ GPN represents $0.2 \%-1.3 \%$ of all facial pain syndromes. ${ }^{3,4}$

The first-line treatment for GPN is medical suppression using agents such as carbamazepine, phenytoin, and gabapentin. ${ }^{11,18}$ If medical treatment has failed or side effects have occurred, surgical treatment should be considered. Microvascular decompression (MVD) and sectioning of the glossopharyngeal nerve are associated with high rates of pain relief at the expense of persistent lower cranial nerve dysfunction. ${ }^{4,13,15}$

Stereotactic radiosurgery (SRS) has been proposed as a minimally invasive alternative management option for GPN. $6,9,10,12,14,20-23$ The International Gamma Knife Research Foundation (IGKRF) was established to evaluate outcomes of selected clinical indications that are relatively rare and to facilitate prospective clinical trials. This report is based on retrospective data provided by 6 academic medical centers that participate in the IGKRF.

\section{Methods \\ Patient Selection}

Six academic medical centers, with individual internal review board approvals for retrospective clinical outcome studies, participated: University of Pittsburgh Medical Center (2 patients), Hospital Na Homolce, Prague, Czech Republic (9 patients), Mayo Clinic ${ }^{14}$ (6 patients), Cleveland Clinic (2 patients), University of Sherbrooke ${ }^{20}$ (1 patient), and University of Virginia ${ }^{22}$ (2 patients). The records of these 22 patients (who underwent SRS between 1998 and 2015) were assessed by each center for inclusion. All data were de-identified and submitted to the IGKRF clinical coordinating center at the University of Pittsburgh Medical Center. Patients with secondary GPN related to tumors, aneurysms, infections, or multiple sclerosis were excluded. Clinical outcomes were assessed at a median follow-up of 45 months (range 6-120 months). Assessment was performed during outpatient visits or by telephone interviews conducted by clinical staff at each institution.

Prior to SRS, all patients underwent clinical assessment, neurological examination, and imaging studies. All patients had repeated episodes of sharp and stabbing pain in the tongue, throat, ear, and tonsils, which lasted from a few seconds to a few minutes. The pain occurred many times each day and could sometimes be triggered by chewing, coughing, laughing, swallowing, and speaking. MRI or CT studies were performed to detect neurovascular compression, demyelinating disease, or cerebellopontine angle or sphenopalatine lesions. All patients were intolerant or refractory to comprehensive medical therapy.

\section{Clinical Material}

This series included 14 women (64\%) and 8 men (36\%). The median age of patients was 60 years (range 34-83 years). The median interval between initial pain onset and SRS was 46 months (range 1-240 months). Patient demographic data are shown in Table 1. Three patients had unsuccessful prior surgical procedures, including MVD (n $=2 ; 14$ months and 50 months before SRS, respectively) and balloon compression ( $\mathrm{n}=1 ; 3$ months before SRS).
TABLE 1. Patient demographic data and SRS characteristics

\begin{tabular}{|c|c|}
\hline Characteristic & No. or Median (range) \\
\hline Male & 8 \\
\hline Female & 14 \\
\hline Median age in yrs & $60(34-83)$ \\
\hline \multicolumn{2}{|l|}{ Prior failed surgical management } \\
\hline MVD (14 mos \& 50 mos before SRS) & 2 \\
\hline Balloon compression (3 mos before SRS) & 1 \\
\hline \multicolumn{2}{|l|}{ Prior failed medication or nerve block } \\
\hline Medication & 18 \\
\hline Nerve block (17 mos before SRS) & 1 \\
\hline \multicolumn{2}{|l|}{ Imaging for SRS planning } \\
\hline MRI alone & 13 \\
\hline MRI \& CT & 9 \\
\hline Neurovascular compression on MRI & 15 \\
\hline Interval btwn initial pain \& SRS in mos & $46(1-240)$ \\
\hline \multicolumn{2}{|l|}{ Duration of pain for each event } \\
\hline $0-30 \mathrm{sec}$ & 9 \\
\hline $31-60 \mathrm{sec}$ & 6 \\
\hline$>1 \min$ & 7 \\
\hline \multicolumn{2}{|l|}{ Pain location } \\
\hline Throat & 18 \\
\hline Tongue & 8 \\
\hline Ear & 14 \\
\hline Median max dose in Gy & $80(80-90)$ \\
\hline Collimator size in $\mathrm{mm}$ & 4 \\
\hline No. of isocenters* & 1 \\
\hline Median clinical follow-up in mos & $45(6-120)$ \\
\hline
\end{tabular}

* In 1 patient, 2 isocenters were used to irradiate a longer nerve segment.

One patient underwent peripheral nerve block 17 months before SRS (Fig. 1). The pain was left-sided in 15 patients and right-sided in 7 patients. The pain duration for each event was 0-30 seconds in 9 patients, 31-60 seconds in 6 patients, and $>1$ minute in 7 patients. The pain location included throat in 18 patients, ear in 14 patients, and tongue in 8 patients.

\section{Radiosurgical Procedure}

The models B, C, 4C, and Perfexion Gamma Knife units were used for SRS, depending on the technology available at participating centers. After application of the Leksell model G stereotactic frame (Elekta Instruments) while patients were under local anesthesia, usually supplemented by mild intravenous sedation, all patients underwent stereotactic MRI to identify the glossopharyngeal nerve. The MR studies were performed using contrastenhanced, short-repetition time sequences and axial volume acquisitions of $512 \times 216$ matrices divided into 1-mm slices. When the glossopharyngeal nerve was difficult to identify on images (usually because of previous surgery), additional axial long-relaxation time MR images were obtained. A single 4-mm isocenter was used for targeting the glossopharyngeal nerve at the level of the glossopharyngeal meatus (Fig. 2). In 1 patient, 2 isocenters were used to 


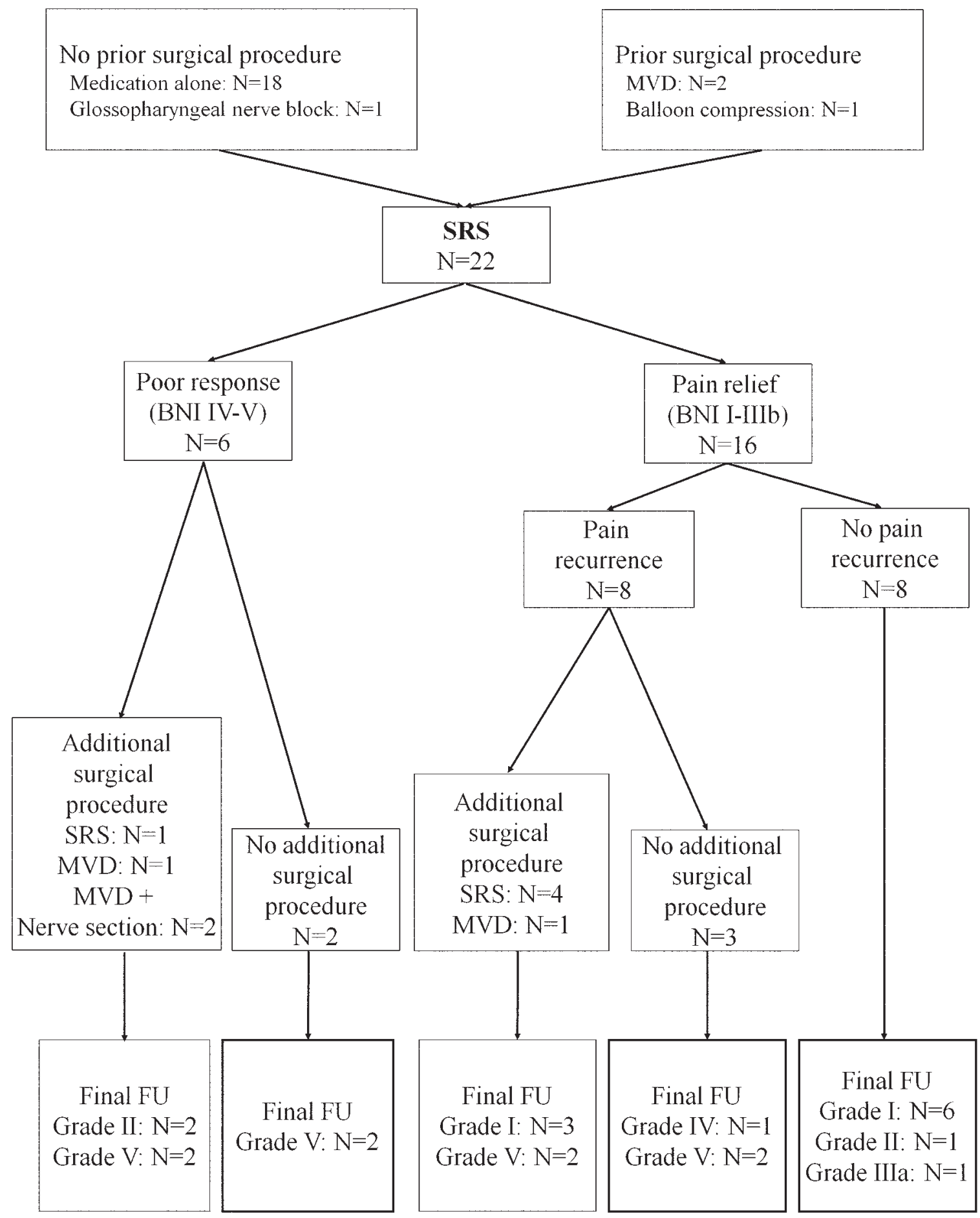

FIG. 1. Flow chart of patients' prior treatments, post-SRS modalities, and outcomes. FU = follow-up.

irradiate a longer nerve segment. The median maximum dose was 80 Gy (range 80-90 Gy). A team consisting of a neurosurgeon, radiation oncologist, and medical physicist performed dose selection and planning.

\section{Evaluation Criteria}

All serial follow-up information was obtained from the patients or their referring physicians and included the de- gree of pain relief, latency interval to pain relief, need for further surgical procedures, use of medication, and new symptoms. In addition, up-to-date clinical information was obtained for all patients by telephone interviews conducted by physicians who were not involved in treatment. Clinical results were classified according to the Barrow Neurological Institute (BNI) pain intensity score: Grade I (pain free, no use of medication), Grade II (occasional pain but off 

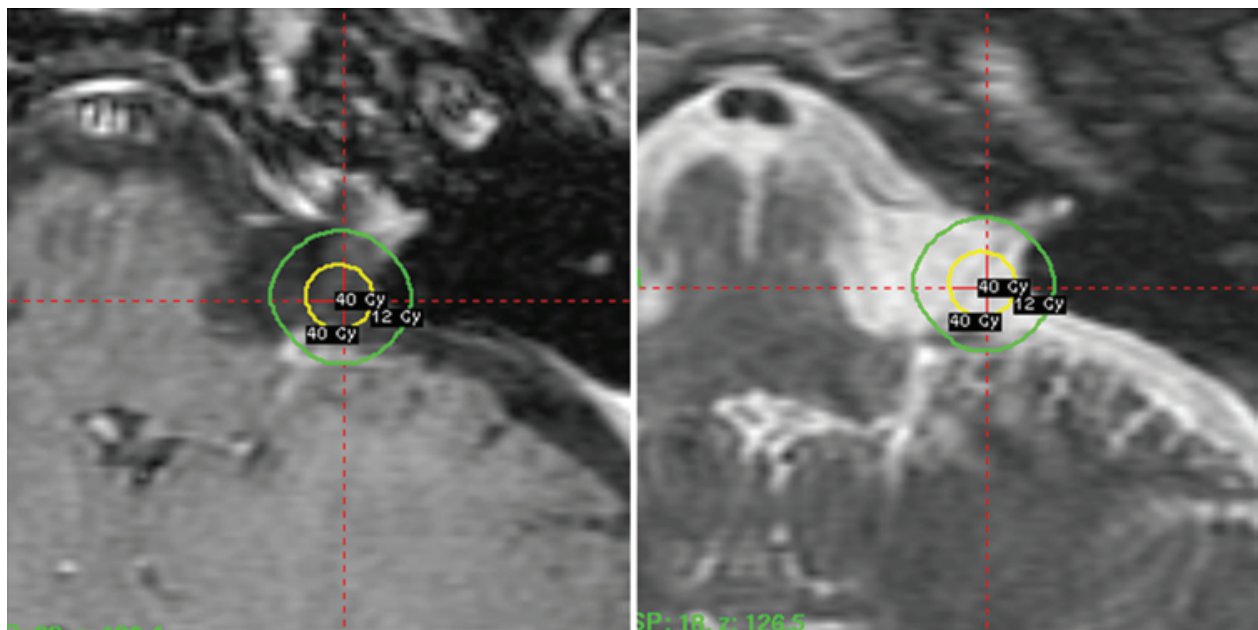

FIG. 2. The radiosurgery dose plan was designed using axial T1-weighted contrast-enhanced (left) and 3D T2-weighted (right) MR images. The dose plan shows 40-Gy (yellow) and 12-Gy (green) isodose lines on the glossopharyngeal meatus of the jugular foramen. The maximum dose is $80 \mathrm{~Gy}$.

of medication), Grade IIIa (no pain and continued use of medications required), Grade IIIb (some pain, controlled with medication), Grade IV (pain improved but not adequately controlled with medication), and Grade V (no pain relief). We defined BNI Grade I as representing complete pain relief and BNI Grades I-IIIb as representing adequate pain relief, whereas BNI Grades IV and V were defined as treatment failures. ${ }^{17}$ Recurrence was defined as a return to a preradiosurgery clinical state and grade in patients who had reached Grades I-IIIb outcomes after SRS. The time to response after radiosurgery was analyzed using the product-limit method of Kaplan and Meier.

\section{Results}

\section{Initial Pain Relief}

Eleven patients $(50 \%)$ had initial complete pain relief (BNI Grade I). The initial complete pain relief rate was $41 \%$ at 1 month, 52\% at 2 months, and 52\% at 3 months (Fig. 3 upper). Sixteen patients (73\%) had initial adequate pain relief (BNI Grades I-IIIb). The initial adequate pain relief rate was $50 \%$ at 1 month, $64 \%$ at 2 months, and $73 \%$ at 3 months (Fig. 3 lower). Eleven patients had BNI Grade I, 2 had Grade II, 1 had Grade IIIa, and 2 had Grade IIIb outcomes after SRS. Six patients (27\%) had no initial pain relief (BNI Grade V). A single patient who had no pain relief underwent subsequent MVD 5 months after SRS without relief. Two patients who had no pain relief underwent MVD followed by nerve section 2-8 months after SRS and achieved adequate pain relief (BNI Grade II). One patient without initial pain relief failed to obtain pain relief despite a second SRS 7 months after the initial procedure. This patient developed hypesthesia in the palatoglossal arch consistent with adverse effects of radiation 5 months after repeat SRS. Two patients remained BNI Grade V but underwent no additional surgical managements at the time of final follow-up. There were no factors (age, sex, time to diagnosis prior to SRS, prior surgical management, margin dose, pain location, and lasting time of pain for each event) statistically related to initial pain relief.

\section{Maintenance of Pain Relief}

Complete pain relief (BNI Grade I) was maintained in 7 patients. The actuarial rates of maintenance of complete pain relief were $59 \%$ at 1 year, $44 \%$ at 2 years, $33 \%$ at 3 years, $33 \%$ at 5 years, and 22\% at 7 years (Fig. 4 upper). Adequate pain relief (BNI Grades I-IIIb) was maintained in 8 patients ( 7 Grade I and 1 Grade IIIa) at the time of final follow-up. The actuarial rates of maintenance of adequate pain relief were $63 \%$ at 1 year, $49 \%$ at 2 years, $38 \%$ at 3 years, $38 \%$ at 5 years, and $28 \%$ at 7 years (Fig. 4 lower). When 7 patients without pain recurrence within 4 years of follow-up were excluded, the rates of maintenance of adequate pain relief were $38 \%$ at 5 years and $28 \%$ at 7 years. There were no factors (age, sex, time to diagnosis prior to SRS, prior surgical management, margin dose, pain location, and lasting time of pain for each event) statistically related to the maintenance of complete or adequate pain relief.

\section{Additional Surgical Management After Initial SRS}

Eight of 16 patients who had initial adequate pain relief suffered pain recurrence. Four patients underwent repeat SRS (maximum dose 75-85 Gy) for the glossopharyngeal meatus at a median of 32 months after initial SRS (range 19-70 months). Two patients maintained complete pain relief at 12 and 18 months after repeat SRS. One patient had initial pain relief but had a pain recurrence 50 months after repeat SRS. One patient had no follow-up after repeat SRS. One patient underwent MVD 65 months after the initial SRS and obtained complete pain relief. One patient underwent peripheral nerve blocks with transient benefit.

\section{Morbidity of Radiosurgery}

Two patients developed persistent hypesthesia in the palatoglossal arch 5 and 8 months after repeat SRS, respectively. Both patients were given the maximum dose of $80 \mathrm{~Gy}$ at both the initial and the repeat SRS. Their complete pain relief was maintained for 87 months and 33 months after repeat SRS, respectively. No patient de- 

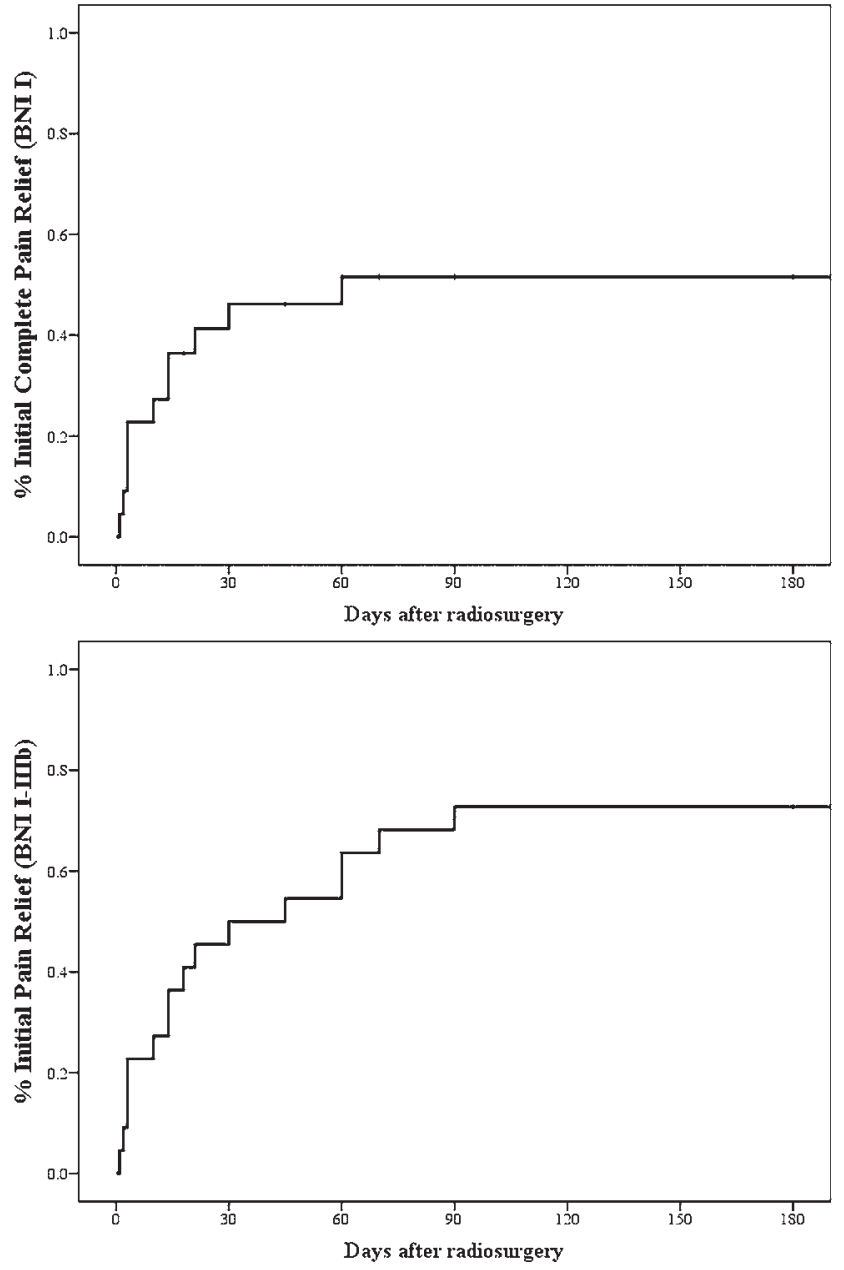

FIG. 3. Kaplan-Meier curves: estimate of probability of initial pain relief after SRS. Upper: Initial complete pain relief of BNI Grade I. Lower: Initial favorable pain relief of BNI Grades I-IIlb.

veloped changes in vocal cord function or swallowing disorders. No patient developed aspiration. No morbidity occurred in patients who underwent a single SRS.

\section{Discussion}

Glossopharyngeal neuralgia is an episodic and debilitating craniofacial pain syndrome similar to trigeminal neuralgia $(\mathrm{TN})$ but affecting sensory distribution of the throat and ear. The pain usually lasts from a few seconds to a few minutes. In the present study, the duration of episodic GPN pain was $>1$ minute in 15 of 22 patients. The pain attacks related to GPN are typically described as occurring in the posterior tongue, throat, pharynx, and ear. Secondary GPN related to adjacent tumor or infection is more common than idiopathic GPN, which is the focus of the small outcome study presented in this report. ${ }^{2}$

The management strategy for GPN is similar to TN. Surgical management is considered in patients who are refractory to medical treatment. The surgical management options for GPN include both destructive and nondestructive procedures. The sectioning (or rhizotomy) of the glossopharyngeal nerve is a potentially effective management
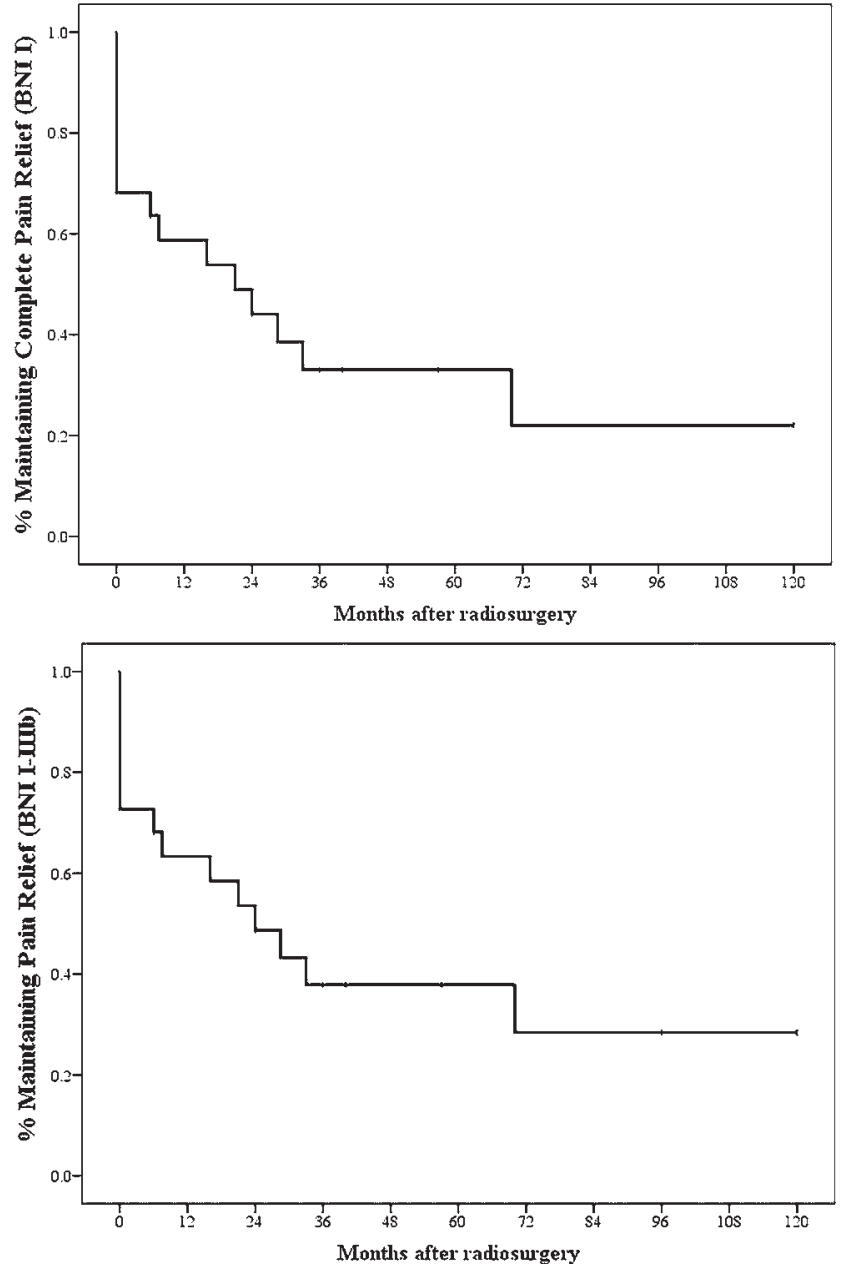

FIG. 4. Kaplan-Meier curves: estimate of maintaining pain relief after SRS. Upper: Maintaining complete pain relief of BNI Grade I. Lower: Maintaining favorable pain relief of BNI Grades I-IIlb.

option, but it is associated with a high rate of postoperative hoarseness and dysphasia. MVD for GPN is a nondestructive surgical management option with reported rates of complete pain relief of $76 \%-97 \%$ and low complication rates (3\%-19\%). ${ }^{12,13,15,19}$ Rey-Dios and Cohen-Gadol ${ }^{16}$ reported that the rate of long-term freedom from pain for patients who underwent MVD was $84.7 \%$ and that the recurrence rate was $7 \%$. Patients who underwent rhizotomy experienced long-term pain relief in $87.3 \%$ of cases, with pain recurrence in $8.2 \%$. The surgical mortality rates for MVD and rhizotomy were $1.1 \%$ and 5\%, respectively. Permanent lower cranial nerve deficits occurred in $5.5 \%$ of MVD and in $19.1 \%$ of rhizotomy patients. In the present SRS study, no adverse effects of radiation were observed after a single SRS, but 2 patients developed persistent hypesthesia in the palatoglossal arch after repeat SRS.

Stereotactic radiosurgery has become a well-accepted surgical management option for intractable TN, associated with satisfactory effectiveness and low morbidity rates. ${ }^{7,8}$ We reported on 503 patients with idiopathic TN who underwent SRS. ${ }^{8}$ Complete or partial pain relief was achieved in $89 \%$ of patients at 1 year. Forty-six percent of patients had adequate pain relief at 5 years. Only $11 \%$ 
developed new or increased subjective facial paresthesia or numbness. Recently SRS has been used to treat GPN based on the favorable outcomes of radiosurgery for TN, but experience is limited.

The first report of GPN radiosurgery was by Stieber et al. ${ }^{21}$ in 2005. The SRS target was the cisternal segment of the glossopharyngeal nerve. The maximum dose was 80 Gy, using a single 4-mm collimator. The patient had initial complete pain relief 3 months after SRS, but pain recurred 6 months after SRS. Lévêque et al. ${ }^{9}$ reported on 7 patients who underwent SRS for intractable GPN. The maximum doses varied from 60 to 80 Gy. The first 2 patients, who received either 60 or $70 \mathrm{~Gy}$, achieved initial complete pain relief but eventually deteriorated to BNI Grade IV and required further intervention. Four patients, who received $\geq$ $75 \mathrm{~Gy}$ to the glossopharyngeal meatus of the jugular foramen, achieved initial complete pain relief. Two of them maintained BNI Grade I, and the remaining 2 patients had slight deterioration to BNI Grade II without any further surgical intervention. No adverse effects of radiation were observed. The authors recommended that the dose for achieving effective pain relief in GPN should be $\geq 75$ Gy.

Martínez-Álvarez et al. ${ }^{10}$ reported on 5 patients who underwent SRS for intractable GPN. One patient underwent rhizotomy and 2 patients had MVD before SRS. The maximum dose was 80-90 Gy to the level of the glossopharyngeal meatus of the jugular foramen. All 5 patients achieved initial BNI Grades I $(n=3)$ or II $(n=2)$ at a median of 6 months (range 2-10 months) after SRS and maintained BNI Grades I or II at a mean follow-up of 43 months (range 14-83 months). No adverse effects of radiation were observed.

In the present study, the 3-month initial favorable pain relief (BNI Grade IIIb) was 73\%. The rates of maintaining favorable pain relief (BNI Grades I-IIIb) were 63\% at 1 year, $49 \%$ at 2 years, $38 \%$ at $3-5$ years, and $28 \%$ at 7 years. A total of 11 of 22 patients $(50 \%)$ underwent additional surgical management, including repeat SRS $(n=6)$, MVD $(\mathrm{n}=2)$, MVD plus nerve section $(\mathrm{n}=2)$, and nerve block ( $\mathrm{n}$ $=1$ ). Repeat SRS can be considered in patients who have had a pain recurrence. Eleven of 22 patients $(50 \%)$ maintained complete pain relief (BNI Grade I) and 12 patients (55\%) maintained favorable pain relief (BNI Grades IIIIb) at the final follow-up after 1-2 SRS procedures.

\section{Conclusions}

In the experience of this retrospective multicenter outcome study, SRS for medically intractable GPN eventually provided pain control or improvement in the majority of patients (73\%). It was associated with a risk of throat sensory dysfunction, but no vocal cord weakness after repeat SRS. Lasting pain improvement occurred in 55\% of patients, some of whom required 2 SRS procedures. Further experience is warranted in using this surgical option for patients who are poor candidates for initial MVD or nerve section. A prospective trial using a higher initial dose may be warranted.

\section{References}

1. Barbash GI, Keren G, Korczyn AD, Sharpless NS, Chayen M,
Copperman Y, et al: Mechanisms of syncope in glossopharyngeal neuralgia. Electroencephalogr Clin Neurophysiol 63:231-235, 1986

2. Bullitt E, Tew JM, Boyd J: Intracranial tumors in patients with facial pain. J Neurosurg 64:865-871, 1986

3. Chawla JC, Falconer MA: Glossopharyngeal and vagal neuralgia. BMJ 3:529-531, 1967

4. Chen J, Sindou M: Vago-glossopharyngeal neuralgia: a literature review of neurosurgical experience. Acta Neurochir (Wien) 157:311-321, 2015

5. Ferrante L, Artico M, Nardacci B, Fraioli B, Cosentino F, Fortuna A: Glossopharyngeal neuralgia with cardiac syncope. Neurosurgery 36:58-63, 1995

6. Héroux F, Mathieu D: Treatment of glossopharyngeal neuralgia by Gamma Knife radiosurgery. Can J Neurol Sci 42:350-352, 2015

7. Kano H, Kondziolka D, Yang HC, Zorro O, Lobato-Polo J, Flannery TJ, et al: Outcome predictors after gamma knife radiosurgery for recurrent trigeminal neuralgia. Neurosurgery 67:1637-1645, 2010

8. Kondziolka D, Zorro O, Lobato-Polo J, Kano H, Flannery TJ, Flickinger JC, et al: Gamma Knife stereotactic radiosurgery for idiopathic trigeminal neuralgia. J Neurosurg 112:758765,2010

9. Lévêque M, Park MC, Melhaoul A, Yomo S, Donnet A, Régis J: Gamma Knife radiosurgery for glossopharyngeal neuralgia: Marseille experience. J Radiosurg SBRT 1:4146, 2011

10. Martínez-Álvarez R, Martínez-Moreno N, Kusak ME, ReyPortolés G: Glossopharyngeal neuralgia and radiosurgery. J Neurosurg 121 Suppl:222-225, 2014

11. Moretti R, Torre P, Antonello RM, Bava A, Cazzato G: Gabapentin treatment of glossopharyngeal neuralgia: a follow-up of four years of a single case. Eur J Pain 6:403-407, 2002

12. O'Connor JK, Bidiwala S: Effectiveness and safety of Gamma Knife radiosurgery for glossopharyngeal neuralgia. Proc (Bayl Univ Med Cent) 26:262-264, 2013

13. Patel A, Kassam A, Horowitz M, Chang YF: Microvascular decompression in the management of glossopharyngeal neuralgia: analysis of 217 cases. Neurosurgery 50:705-711, 2002

14. Pollock BE, Boes CJ: Stereotactic radiosurgery for glossopharyngeal neuralgia: preliminary report of 5 cases. J Neurosurg 115:936-939, 2011

15. Resnick DK, Jannetta PJ, Bissonnette D, Jho HD, Lanzino G: Microvascular decompression for glossopharyngeal neuralgia. Neurosurgery 36:64-69, 1995

16. Rey-Dios R, Cohen-Gadol AA: Current neurosurgical management of glossopharyngeal neuralgia and technical nuances for microvascular decompression surgery. Neurosurg Focus 34(3):E8, 2013

17. Rogers CL, Shetter AG, Fiedler JA, Smith KA, Han PP, Speiser BL: Gamma Knife radiosurgery for trigeminal neuralgia: the initial experience of The Barrow Neurological Institute. Int J Radiat Oncol Biol Phys 47:1013-1019, 2000

18. Rozen TD: Trigeminal neuralgia and glossopharyngeal neuralgia. Neurol Clin 22:185-206, 2004

19. Sampson JH, Grossi PM, Asaoka K, Fukushima T: Microvascular decompression for glossopharyngeal neuralgia: long-term effectiveness and complication avoidance. Neurosurgery 54:884-890, 2004

20. Stanic S, Franklin SD, Pappas CT, Stern RL: Gamma Knife radiosurgery for recurrent glossopharyngeal neuralgia after microvascular decompression. Stereotact Funct Neurosurg 90:188-191, 2012

21. Stieber VW, Bourland JD, Ellis TL: Glossopharyngeal neuralgia treated with Gamma Knife surgery: treatment outcome and failure analysis. Case report. J Neurosurg 102 Suppl:155-157, 2005

22. Williams BJ, Schlesinger D, Sheehan J: Glossopharyngeal 
neuralgia treated with Gamma Knife radiosurgery. World Neurosurg 73:413-417, 2010

23. Yomo S, Arkha Y, Donnet A, Régis J: Gamma Knife surgery for glossopharyngeal neuralgia. J Neurosurg 110:559-563, 2009

\section{Disclosures}

Dr. Kano received clinical or research support for this study (includes equipment or material) from Elekta AB. The work described in this report was funded by a grant (to H.K.) from the Elekta Research Grant. Dr. Lunsford is a consultant for Elekta $\mathrm{AB}$, has direct stock ownership in Elekta $\mathrm{AB}$, and is a consultant for DSMB and Insightec.

\section{Author Contributions}

Conception and design: Kano. Acquisition of data: Kano,

Urgosik, Liscak, Pollock, Cohen-Inbar, Sheehan, Sharma, Silva,
Barnett, Mathieu, Sisterson. Analysis and interpretation of data: Kano. Drafting the article: Kano, Lunsford. Critically revising the article: all authors. Reviewed submitted version of manuscript: all authors. Approved the final version of the manuscript on behalf of all authors: Kano.

\section{Supplemental Information}

Previous Presentations

This paper was presented at the 18th International Leksell

Gamma Knife Society Meeting, Amsterdam, The Netherlands, May 15-19, 2016.

\section{Correspondence}

Hideyuki Kano, Department of Neurological Surgery, University of Pittsburgh, Ste. B-400, University of Pittsburgh Medical Center Presbyterian, 200 Lothrop St., Pittsburgh, PA 15213. email: kanoh@upmc.edu. 